\title{
Robust Active Contour Model Guided by Local Binary Pattern Stopping Function
}

\author{
Abdallah Azizi $i^{1,3}$, Kaouther Elkourd ${ }^{2,3}$, Zineb Azizi ${ }^{1}$ \\ ${ }^{1}$ Department of Electrical Engineering, University of Mohamed Khider, B.P 145 RP, Biskra, Algeria \\ ${ }^{2}$ Department of Physics, University of Ben Youcef Ben Khedda, Algiers, Algeria \\ ${ }^{3}$ Laboratory of Identification, Command, Control and Communication "LI3CUB", University of \\ Mohamed Khider, B.P 145 RP, Biskra, Algeria \\ E-mails: aziziabdallah87@yahoo.com Kaouther_youcef@yahoo.fr \\ blackblossom@rocketmail.com
}

Abstract: Edge based active contour models are adequate to some extent in segmenting images with intensity inhomogeneity but often fail when applied to images with poorly defined or noisy boundaries. Instead of the classical and widely used gradient or edge stopping function which fails to stop contour evolution at such boundaries, we use local binary pattern stopping function to construct a robust and effective active contour model for image segmentation. In fact, comparing to edge stopping function, local binary pattern stopping function accurately distinguishes object's boundaries and determines the local intensity variation dint to the local binary pattern textons used to classify the image regions. Moreover, the local binary pattern stopping function is applied using a variational level set formulation that forces the level set function to be close to a signed distance function to eliminate costly re-initialization and speed up the motion of the curve. Experiments on several gray level images confirm the advantages and the effectiveness the proposed model.

Keywords: Active contour models, edge stopping function, image segmentation, local binary pattern.

\section{Introduction}

Image segmentation is one of the most studied problems in the field of computer vision. There exist many approaches to tackle this problem, such as region merging [1], region growing [2] and Active Contour Models (ACMs) [3-11]. The idea of ACM is to drive a curve from a given image to reach the boundaries of the interested objects by minimizing energy, where the curve is the argument of this energy.

According to curve representation, there are two types of ACM, parametric models and geometric models. Parametric ACM [3-5] are implemented explicitly as 
parameterized curves with a set of control points, and evolving the curve is reached by evolving these control points. However, by construction, parametric curves have a fixed topology; without additional splitting or merging heuristics, the curve topology will not change during its evolution. Geometric ACM are independently introduced by $\mathrm{Casselles}$ et al. [6], Caselles, Kimmel and Sapiro [7] and Malladi, Sethian and Vemuri [8]. These models combining curve evolution theory [12] and Level Set Method (LSM) [13], give sophisticated solution to overcome the limitation of parametric ACM. The key idea is to evolve a Level Set Function (LSF) where the curve is representing its zero level. The main advantage of this approach is that the topological changes of the curve represented by the LSF are handled automatically.

According to the LSM strategy, there are two different strategies to tackle segmentation with LSM: Partial Differential Equation (PDE) based ones [3, 6-9] and variational ones $[10,11]$. The Level Set Evolution (LSE) of PDE based LSM is directly derived by implementing the gradient descent equation for the curve using the level set equation. This was done to derive the LSM for snake-like energies known as Geodesic Active Contour (GAC) [7, 9]. The LSE of variational LSM is derived by rewriting the variational principle with respect to the LSF rather than the curve, and then computing the gradient descent with respect to this function $[10,11]$. This method presents advantages over PDE based one, that it is more robust and more convenient for incorporating additional information such as shape and region location [14].

According to the energy, ACMs are classified into two main categories: edge based models and region based models. Edge based models $[7,10]$ rely on local image gradient information to construct an Edge Stopping Function (ESF) to stop the evolving curve on the desired objects boundaries. Although these models have been successfully applied for images with height variation in gradient at objects boundaries even in the presence of intensity inhomogeneity, they meet difficulty when dealing with object having blurred or discrete boundaries and they hardly detect objects corrupted by noise [15]. Region based models form the foreground and background regions statistically and find an energy optimum where the model best fits the image [16]. These models control the evolution of the curve with better performance including weak edge and noise. However, modelling regions using global statistics $[11,17]$ is not applicable to images with intensity inhomogeneity. Although, some region based models using local statistics have been proposed to deal with intensity inhomogeneity [18-20] but they are found to act locally, to be easy to trap into local minima and the local statistics are only defined empirically, which make these models sensitive to the choice of those statistics [21].

To improve the performance of ACMs, some hybrid models were proposed by combining either edge and global region information [22] or local and global region information [23]. In such models, edge and local region information are suitable in regions with intensity inhomogeneity, while global region information is favored in noisy and flat regions. The competition between the combined information is controlled by a weighting parameter that needed to be chosen appropriately. 
In implementing the traditional LSM, the LSF is initialized to be a Signed Distance Function (SDF) and for numerical stability, it is necessary to keep the evolving LSF close to a SDF. A remedy procedure called Re-initialization is applied periodically during the evolution of the LSF to keep the property of a SDF. However, it is very time consuming [24].

Recently, Li et al. [10] proposed a variational level set formulation which regularizes the LSF during its evolution by forcing it to be close to a SDF and therefore the costly re-initialization procedure can be eliminated. This variational level set formulation has many advantages over the traditional LSF formulations including higher efficiency and easier implementation [25]. However, since the model proposed in [10] is an edge based ACM and relies on ESF, this leads back to drawbacks described above.

To solve problems met with ESF based on gradient information, we propose a stopping function based on the Local Binary Pattern (LBP) textons [26, 27]. This new function is called LBP Stopping Function (LBPSF). The LBP textons was originally used to perform adaptive gradient calculation for edge detection [28] in which, the LBP textons is used to classify regions of an image around a pixel into noisy, homogenous and edge regions, and then, accordingly, an adaptive filter is introduced while calculating the gradient.

The classification of the image regions into: Noisy, homogeneous and edge regions using the LBP textons helps to generate a filter rejecting pixel positions of LBPs which are likely to be produced by noise and calculate gradient magnitudes at the accepted pixel positions to detect the edges using a modified canny edge detector based on LBP, and then, constructing a LBPSF which takes values close to 1 on noisy and homogeneous regions, while having values close to 0 on edges. Consequently, the curve will stop at these edges. Substituting the ESF based gradient information of the variational formulation of the ACM proposed in [10] with the proposed LBPSF; we obtain a fast ACM which is robust to the leakage and noise problems.

\subsection{Related work}

Among the most recent and relevant ACMs using the level set formalism, and as a consequence depending on the kind of the information used, we can mention the edge based model proposed in [10], the global region based model proposed in [17], the local region based model proposed in [21] and the hybrid models proposed in [22] and [23].

In [10], the authors proposed a level set method termed as Distance Regularized Level Set Evolution (DRLSE). This model provides a simple and efficient narrowband implementation without re-initialization [29] by introducing a signed distance penalizing energy functional that measures the closeness between a LSF and a SDF. However since this model is an edge based ACM and relies on an ESF, the curve will either evolve and deviate from the object boundaries in the regions with weak or without edges, or stop before reaching object's boundaries in textured and noisy regions. 
In [17] a region based ACM was proposed. This model benefits from the advantage of the ACM proposed in [30] by introducing a new region based Signed Pressure Force (SPF) using global statistical information inside and outside the curve into a simple and efficient level set updating formulation by using directly the current LSF. Also, a simple morphological opening and closing operation was applied to regularize the LSF and avoid the costly re-initialization. Overall this model is fast, computationally more efficient and parameter free. However, it suffers from low performance on images with several intensity levels or images with intensity inhomogeneity.

In order to deal with images with intensity inhomogeneity, several authors have introduced the local image information instead of global ones in the associated level set formulation [18-20]. However, such models are found to act locally and to be sensitive to initial contour location.

Recently, a local model is proposed in [21] with the aim that can handle intensity inhomogeneity and robust to initial contour location. This model is conceived to be applicable for both simultaneous segmentation and bias correction, in which the inhomogeneous objects are modelled as Gaussian distributions of different means and variances. Due to this fact, the model is able to combine the information about the special dependencies between pixels belonging to the same class and yield a soft segmentation. Besides that, a method based on Gaussian filtering was introduced to regularize the LSF to avoid re-initialization. However, this model is characterized by a high computational cost and to the limitation of relying on a particular probabilistic model [31].

In [22] and [23] the authors considered to combine both boundary information with global region information and local intensity information with global intensity information, respectively. When the contour is far away from object boundaries, the force from the global intensity information is dominant and has large capture range. When the contour is close to the object boundaries, the force from the local intensity information or from boundary information becomes dominant, which attracts the contour toward and finally stops the contour at object boundaries [19]. Combining different information in one model raises the performance of ACM by adding their advantages. But the segmentation result is dependent to the choice of the weighting parameter between the combined information.

The rest of this paper is organized as follow: Section 2 presents a theoretical background of the LBP and introduces the derivation of the LBPSF and the proposed ACM based on it. Section 3 provides experimental results and comparisons to the models proposed in $[10,17,21,22,23]$. Finally, Section 4 concludes the paper.

\section{The proposed method}

In this section, we will introduce the proposed model based LBP; starting by giving a theoretical background about the derivation of the LBP, moving to presenting the edge detection method with LBP proposed in [28] that been based on to construct the LBPSF and finishing by deriving the energy formula of the proposed model. 


\subsection{Local binary pattern}

The LBP operator was first introduced by $\mathrm{Ojala}$, Pietikäinen and Mäenpää [26] and Mäen pää [27] for texture analysis. It has been applied in many active studies such as texture classification and face recognition [32]. The LBP operator combines characteristics of statistics and structural texture analysis; it describes the texture with primitives called textons [33].

The derivation of an LBP code is shown in Fig. 1a; taking a neighbourhood of $3 \times 3$ of a central pixel, thresholding it into two levels " 0 " or " 1 " whether the neighbour of that pixel has smaller or larger value than the central pixel, respectively. An LBP code is obtained by multiplying the threshold values of eight pixels by binomial weights and summing up the result. This leads to the equation

$$
\operatorname{LBP}_{P, R}=\sum_{p=0}^{P-1} s\left(g_{p}-g_{c}\right) 2^{p},
$$

where $P$ is the number of neighbours, $R$ is the radius, $g_{c}$ corresponds to the gray value of the center pixel, $g_{p}$ are the gray values of surrounding pixels and $s(x)$ is

$$
s(x)= \begin{cases}1, & x \geq 0 \\ 0, & x<0\end{cases}
$$

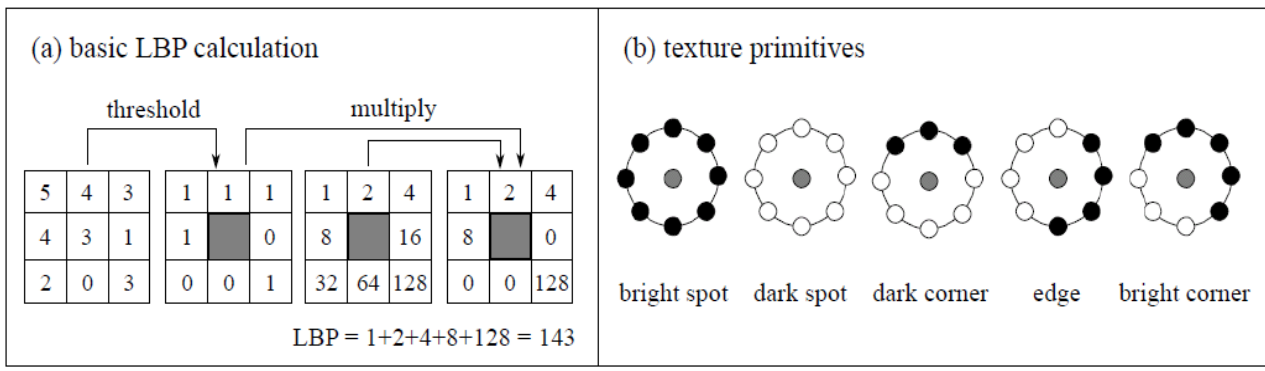

Fig. 1. Calculation and interpretation of Local Binary Patterns (LBPs) [28]

Different texture primitives can be detected by the LBP code, Fig. 1b shows examples where ones and zeros are indicated with white and black circles respectively. A special kind of LBP, which will be used for edge detection, is called rotation-invariant uniform LBP given by:

$$
\mathrm{LBP}_{P, R}^{\text {riu2 }}=\left\{\begin{array}{l}
\sum_{p=0}^{P-1} s\left(g_{p}-g_{c}\right) 2^{p} \text { if } U\left(\mathrm{LBP}_{P, R}\right) \leq 2, \\
P+1 \text { else, }
\end{array}\right.
$$

where $U$ is the number of bitwise $0 / 1$ and $1 / 0$ transitions in an LBP, only two or less are allowed for uniform LBP [28]. Fig. 2 shows the nine classes of the uniform LBP.

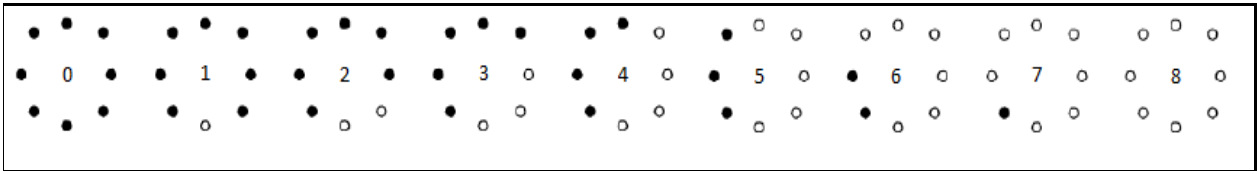

Fig. 2. Classes of the non uniform rotation invariant LBPs 


\subsection{Edge detection with LBP}

This sub-section presents the edge detection method proposed in [28], where the authors introduced a modified Canny edge detector based on LBP. This method is the core element constructing our model in the next sub-section.

For some example images with different levels and types of artificial noise, Teutsch and Beyerer [28] have calculated $\mathrm{LBP}_{P, R}^{\text {riu2 }}$ with variable radius $R$ for each pixel and accumulated in LBP histograms. Each histogram has ten bins: nine for the different $\mathrm{LBP}_{P, R}^{\text {riu2 }}$ equivalence classes, which are displayed in Fig. 2, and one for all other LBPs. From Fig. 3, it can be seen that the number of edges of different orientations $\left(\mathrm{LBP}_{P, R}^{\mathrm{riu} 2}\right.$ classes 2-6) decreases when the noise level increases while other LBPs (classes 0, 1, 7, 8 and 9) are much affected by noise [34].

The main idea in this approach is that all pixels of those classes affected by noise are not considered for gradient calculation in edge detection and then, accordingly, noise can be suppressed. Edge detection using LBPs is based on the Canny's edge detector algorithm with modified steps as follow (Fig. 3).

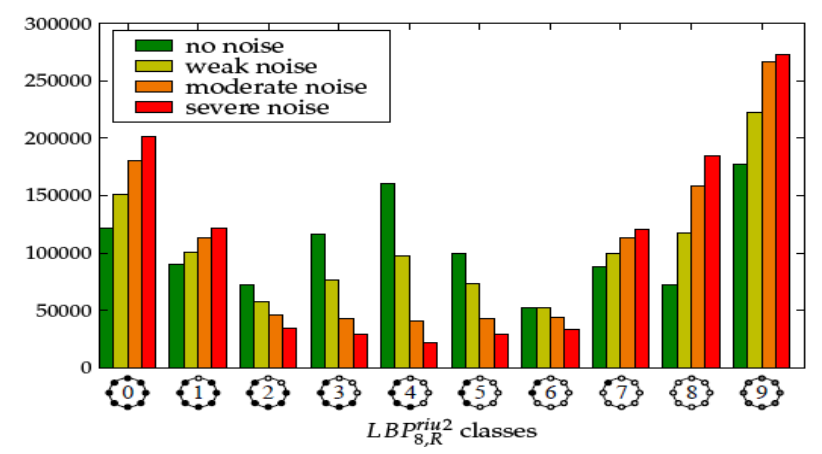

Fig. 3. LBP histogram for Lena with different noise levels [34]

\subsubsection{Noise suppression}

Unlike in Canny's algorithm where noise is suppressed by smoothing with Gaussian kernel, in this approach, a filter is generated which rejects pixel positions of LBPs which are likely to be produced by noise (classes $0,1,7,8$ and 9); a binary function $f$ is applied pixelwise to all image pixel positions $c=(x, y)$ of the following formula:

$$
f(c)=\left\{\begin{array}{l}
1 \text { if } \operatorname{LBP}_{8,1}^{\text {riu2 }} \in \operatorname{classes}(2-6) \\
0 \text { else. }
\end{array}\right.
$$

Only pixel positions with $f(c)=1$ will be considered for the next step.

\subsubsection{Gradient magnitude}

Gradient magnitudes $G(c)$ in this approach are calculated at the accepted pixel positions $(f(c)=1)$ using the local variance $\operatorname{VAR}_{P, R}$ :

$$
G(c)=\left\{\begin{array}{l}
\sum_{r=R_{1}}^{R_{n}} \sqrt{\mathrm{VAR}_{P, r}} \text { if } f(c)=1, \\
0 \text { else, }
\end{array}\right.
$$


where $r$ is the variation of the radius $R$ to calculate several LBPs and summed up for the gradient magnitude in order to increase the robustness against noise. Variance tends to focus too much on bright objects. So, standard-deviation is used instead of variance as it produces more homogeneous edge images [28]:

$$
\operatorname{VAR}_{P, R}=\frac{1}{P} \sum_{p=0}^{P-1}\left(g_{p}-\mu\right)^{2}, \text { where } \mu=\sum_{p=0}^{P-1} g_{p} .
$$

\subsubsection{Non-maximum suppression}

As in Canny's algorithm, four discretized gradient orientations: $0^{\circ}, 45^{\circ}, 90^{\circ}$, and $135^{\circ}$ are used. While these orientations are calculated using the atan 2 function in Canny, this approach doesn't need to calculate anything in order to get the orientation. Instead, four sets: $\mathrm{D}^{0}{ }_{\mathrm{LBP}}, \mathrm{D}^{45}{ }_{\mathrm{LBP}}, \mathrm{D}^{90}{ }_{\mathrm{LBP}}$ and $\mathrm{D}^{135}{ }_{\mathrm{LBP}}$ of $\mathrm{LBP}$ that represent the orientations are simply defined. Each set D consists of $16 \mathrm{LBP}$ as in Fig. 4. Since LBP with an even number of bright/dark dots are ambiguous, we simply say that they belong to two sets [28].

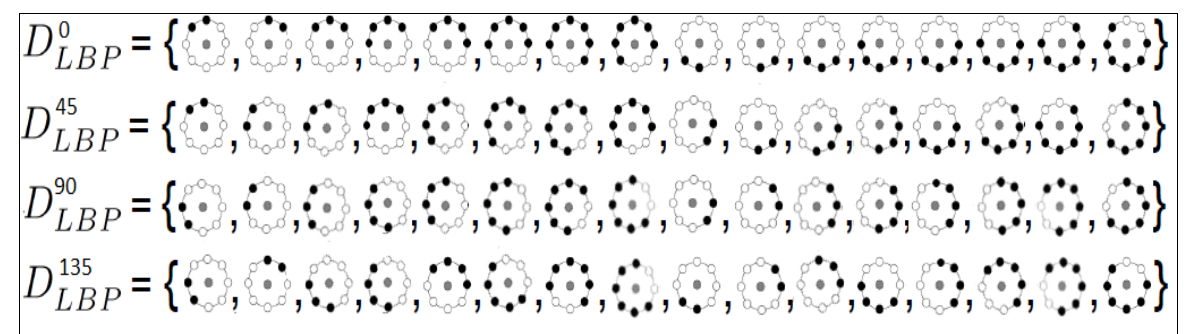

Fig. 4. The four sets with LBPs of different orientations

\subsubsection{Determination of edge pixels}

The final step is to generate a binary edge pixel image $B$ using the hysteresis operator, in which pixels are marked as either edges, non edges and in-between, this is done based on two thresholds $t_{1}$ and $t_{2}$ with $t_{1}<t_{2}$. If a gradient magnitude $G(c)$ exceeds $t_{2}$, it is accepted as edge pixel, while all pixels with gradient value less than $t_{1}$ are marked as non edges. The next step is to consider each of the pixels that are in-between, if they are connected to edge pixels these are marked as edge pixels as well. The result of this edge detector is the binary image $B$ in which the white pixels closely approximate the true edges of the original image.

\subsection{The active contour model based LBP stopping function}

We firstly introduce the LBPSF and to motivate it, we will present the classical ESF and some of its shortcoming.

The philosophy behind the edge-based ACM is finding curve that undergoes strong edges (object boundaries), and to achieve this goal, an external energy is defined that we integrate over the curve to move it toward the object boundaries.

Let $I$ be a given gray level image, the classical ESF is defined as follow:

$$
g(|\nabla I|)=\frac{1}{1+\left|\nabla G_{\sigma} * I\right|^{2}} .
$$


This function assigns small values to strong gradients of the smoothed image $\nabla G_{\sigma} * I$ where the curve is very close to the boundaries. However, these gradient based functions have two major drawbacks:

In practice, the discrete gradients are bounded and then, the function $g$ can be relatively far from zero on the edges and the curve may pass through the boundaries $[11,14]$.

The next issue is that for the noisy or textured regions, the image will have gradient maxima which induce local minima in the external energy. Therefore, the curve will not stop at the real object's boundaries. Alternatively, the Gaussian smoothing is used to remove spurious local minima. Yet, smoothing also removes possibly important edge information if the kernel width $\sigma$ is not chosen appropriately. Hence, based on the edge detection operation with LBP described in the previous sub-section, we construct the LBPSF as follow:

$$
g_{\text {LBP }}=1-B \text {, }
$$

where $B$ is the binary image resulting from the Canny's edge detector with LBP.

The function $g_{\mathrm{LBP}}$ is made such as it is zero on edges whereas it is equal to one on flat and noisy regions and then, accordingly, the active contour will keep evolving in flat and noisy regions till it attains the object boundaries (the edges). Replacing the ESF $g$ in the variational formulation of [10] by the proposed LBPSF $g_{\mathrm{LBP}}$, the total energy function can be defined as

(9) $E(\varphi)=\mu \int_{\Omega} \frac{1}{2}(|\nabla(\varphi)|-1)^{2} d x d y+\lambda \int_{\Omega} g_{\mathrm{LBP}} \delta(\varphi)|\nabla(\varphi)| d x d y+v \int_{\Omega} g_{\mathrm{LBP}} H(-\varphi) d x d y$.

The first term in the right hand side of (9) controlled by $\mu>0$ is called the internal energy term that penalizes the deviation of the LSF $\phi$ from a SDF, whereas the last two terms controlled by $\lambda$ and $v$ form the external energy excerpted from the GAC [7], this energy is defined to drive the motion of the curve toward the object's boundaries. $\delta($.$) and H($.$) are the Dirac function and the Heaviside function,$ respectively. By minimizing (9) with respect of $\phi$, the steepest descent process is the following gradient flow [10]:

$$
\frac{\partial \varphi}{\partial t}=\mu\left[\Delta \varphi-\operatorname{div}\left(\frac{\nabla \varphi}{|\nabla \varphi|}\right)\right]+\lambda \delta(\varphi) \operatorname{div}\left(g_{\mathrm{LBP}} \frac{\nabla \varphi}{|\nabla \varphi|}\right)+v g_{\mathrm{LBP}} \delta(\varphi),
$$

where $\Delta$ and $\operatorname{div}($.$) are the laplacien and the divergence operators, respectively.$

The model in [10] not only eliminates the need of costly re-initialization, but also allows the use of more general initial LSF rather than a SDF initial function, a binary step function is proposed as an initial LSF defined by [10]:

$$
\varphi_{0}(x, y)=\left\{\begin{aligned}
-d & (x ; y) \in \Omega_{0}-\partial \Omega_{0}, \\
0 & (x ; y) \in \partial \Omega_{0}, \\
+d & (x ; y) \in \Omega-\partial \Omega_{0},
\end{aligned}\right.
$$

where $d>0$ is a constant, $\Omega_{0}$ is a subset in the image domain $\Omega$, and $\partial \Omega_{0}$ be all the points in the boundaries of $\Omega_{0}$.

Because of the diffusion introduced by the penalizing energy, there is no need to the upwind scheme as traditional LSM [10]. The partial derivative can be discretized using the forward differences. Thus, (10) is approximated as 


$$
\phi_{i, j}^{n+1}=\Delta t R\left(\phi_{i, j}\right)^{n}+\phi_{i, j}^{n},
$$

where $\Delta t$ is the time step, $\phi_{i, j}^{n}=\phi\left(n \Delta t, x_{i, j}\right)$ is an approximation of $\phi(t, x), x\left(x_{i}, y_{i}\right)$ is the grid points, and $R\left(\phi_{i, j}\right)$ is the approximation of the right hand side in (10).

\section{Results}

In this section, in order to validate the performance of the proposed model, we apply and compare it with the gradient based ACM of [10], the global region based ACM of [17], the local region based ACM proposed in [21] and the hybrid models proposed in [22] and [23] using both synthetic and real gray level images. All models are implemented using Matlab 7.0 in Windows 7; on $3.3 \mathrm{GHz}$ Intel core i3 PC with 4GB of RAM. Unless otherwise specified, the parameters are described in Table 1.

This validation and comparison are performed on a set of 15 gray level images consisting of: a synthetic image with intensity inhomogeneity, a synthetic image with several intensity levels, a fluorescence microscopic image, two noisy ultrasound images of the left ventricle of a human heart, a magnetic resonance images of the same organ, a cardiac CT image, a liver CT image, a radiographic image of weld defect, a ship in a thermal infrared image, a magnetic resonance image of bladder, and four real-world images from the Weizmann segmentation dataset, and the results are illustrated in Fig. 5, Fig. 6 and Fig. 7.

From these results, it is clear that the proposed ACM based LBPSF outperforms the other ACMs in term of efficiency (boundary detection).

In particular, compared the proposed model to the model proposed in [10], no smoothing is necessary for the LBPSF, while the ESF deals with the problem that smoothing with big filter size (Equation (7)) can suppress important edges and the curve will pass through them (Image 1,8 and 13), but small filter size may not be sufficient to remove noise and then, the curve will stop evolving before reaching the real object boundaries (Images: 3-5, 7, 9-12 and 14-15).

Table 1. Description of the parameters used in the study

\begin{tabular}{|c|l|}
\hline Parameters & \\
\hline$d$ & To initialize the LSF, $d>0$ is a constant \\
\hline$\sigma, \rho$ & $\begin{array}{l}\text { Width of the Gaussian kernel in [10], region scale parameter in [21] and [23] } \\
\text { (determined according to images for [21] and } \rho=3 \text { for [23]) }\end{array}$ \\
\hline$\Delta t$ & Time step ([23]: $\Delta t=0.1[17],[21]$ and [22]: $\Delta t=1,[10]$ and our model: $\Delta t=5)$ \\
\hline$\mu$ & Regularization parameter of internal energy ([10] and our model: $\mu=0.04)$ \\
\hline$\lambda, v$ & $\begin{array}{l}\text { Regularization parameters of the curve ([10] and our model: } \lambda=5, v= \pm 3(-) \text { if } \\
\text { initial curve is located inside or }(+) \text { if it is outside) }\end{array}$ \\
\hline$P, R$ & LBP parameters (number of neighbors and LBP radius $(P=8, R=1))$ \\
\hline$t_{1}, t_{2}$ & Threshold parameters determined empirically according to images \\
\hline$\alpha, \beta$ & $\begin{array}{l}\text { Weighting parameters of [22] and [23], respectively (determined empirically } \\
\text { according to images) }\end{array}$ \\
\hline
\end{tabular}




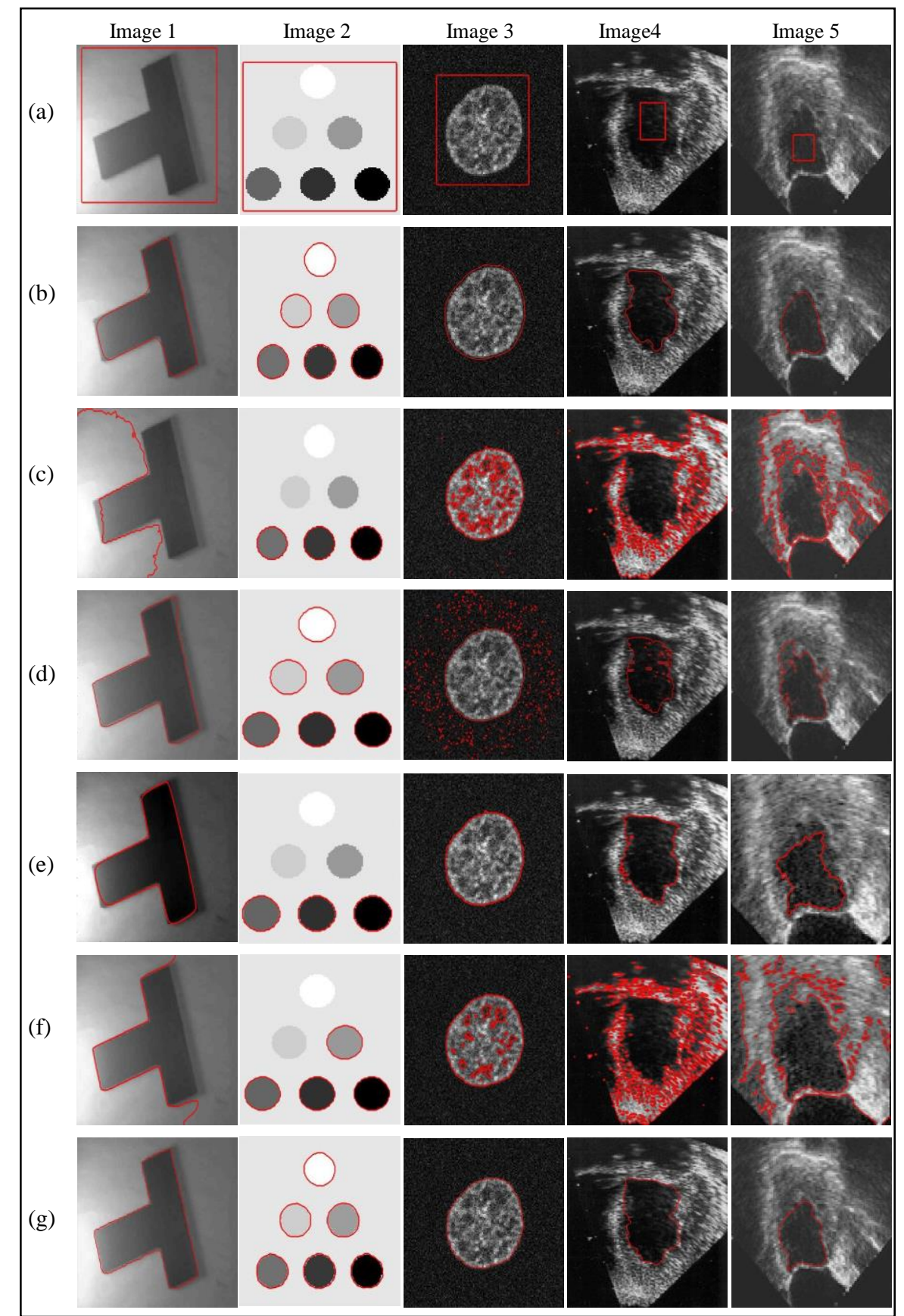

Fig. 5. Comparison between the different active contour models: Original images with initial contours (a). Segmentation output of: Gradient-based model [10] ( $\sigma=1.2$ for all images) (b), global region based model [17] (c), local region based model [21] (Image 1: $\rho=6.0$, Images: 2, 3, and 4: $\rho=3.0$, Image 5: $\rho=1.5)(\mathrm{d})$, the hybrid models $[22,23](\mathrm{e}, \mathrm{f})$ and the proposed model $(\mathrm{g})$ 


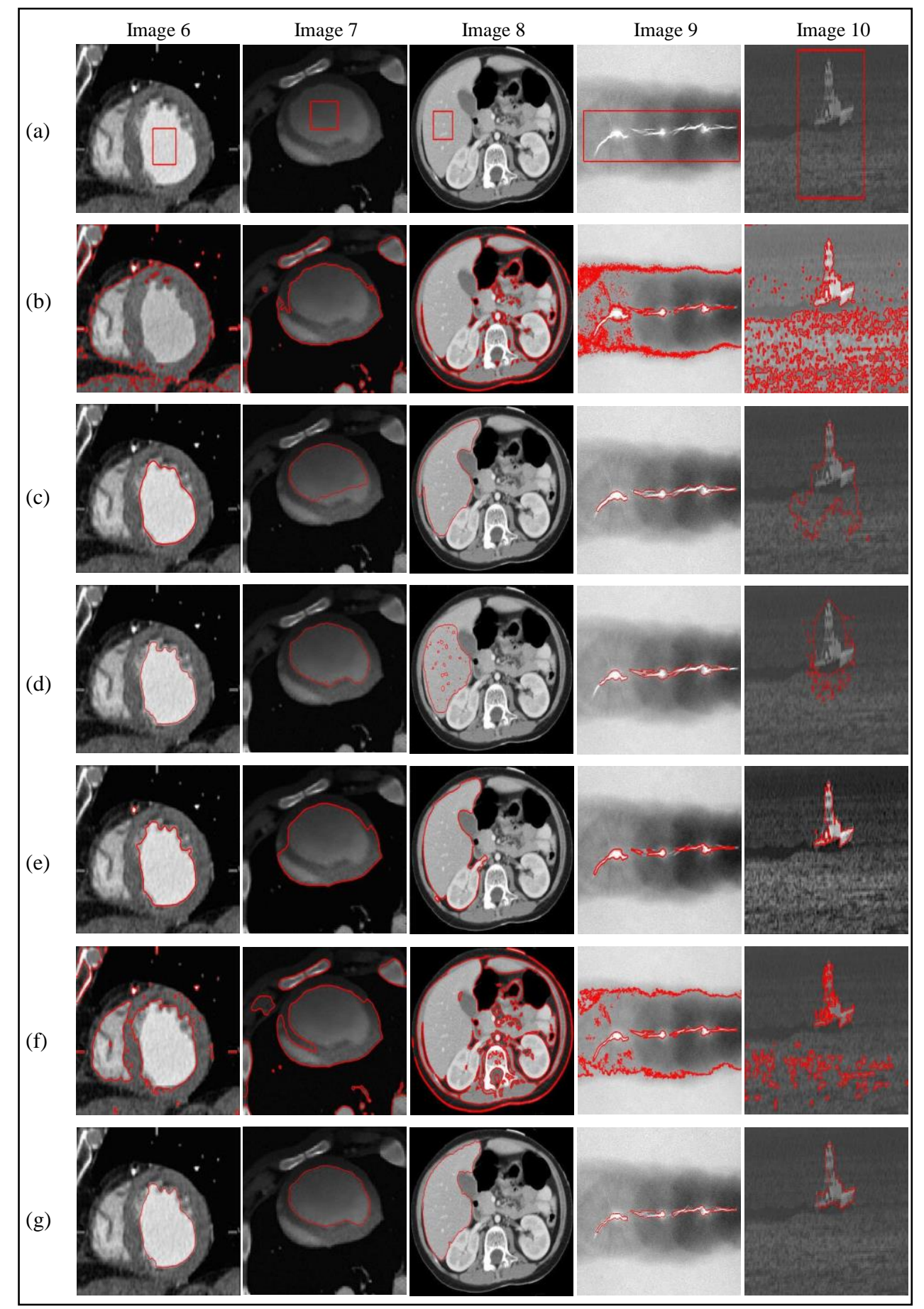

Fig. 6. Comparison between the different active contour models: Original images with initial contours (a). Segmentation output of: Gradient-based model [10] ( $\sigma=4$ for image 8, $\sigma=1.2$ for others) (b), global region based model [17] (c), local region based model [21] (Image 6, 7, and 8: $\rho=3.0$, Image 9: $\rho=6.0$, Image 10: $\rho=4.0)(\mathrm{d})$, the hybrid models [22, 23] (e, f) and the proposed model $(\mathrm{g})$ 


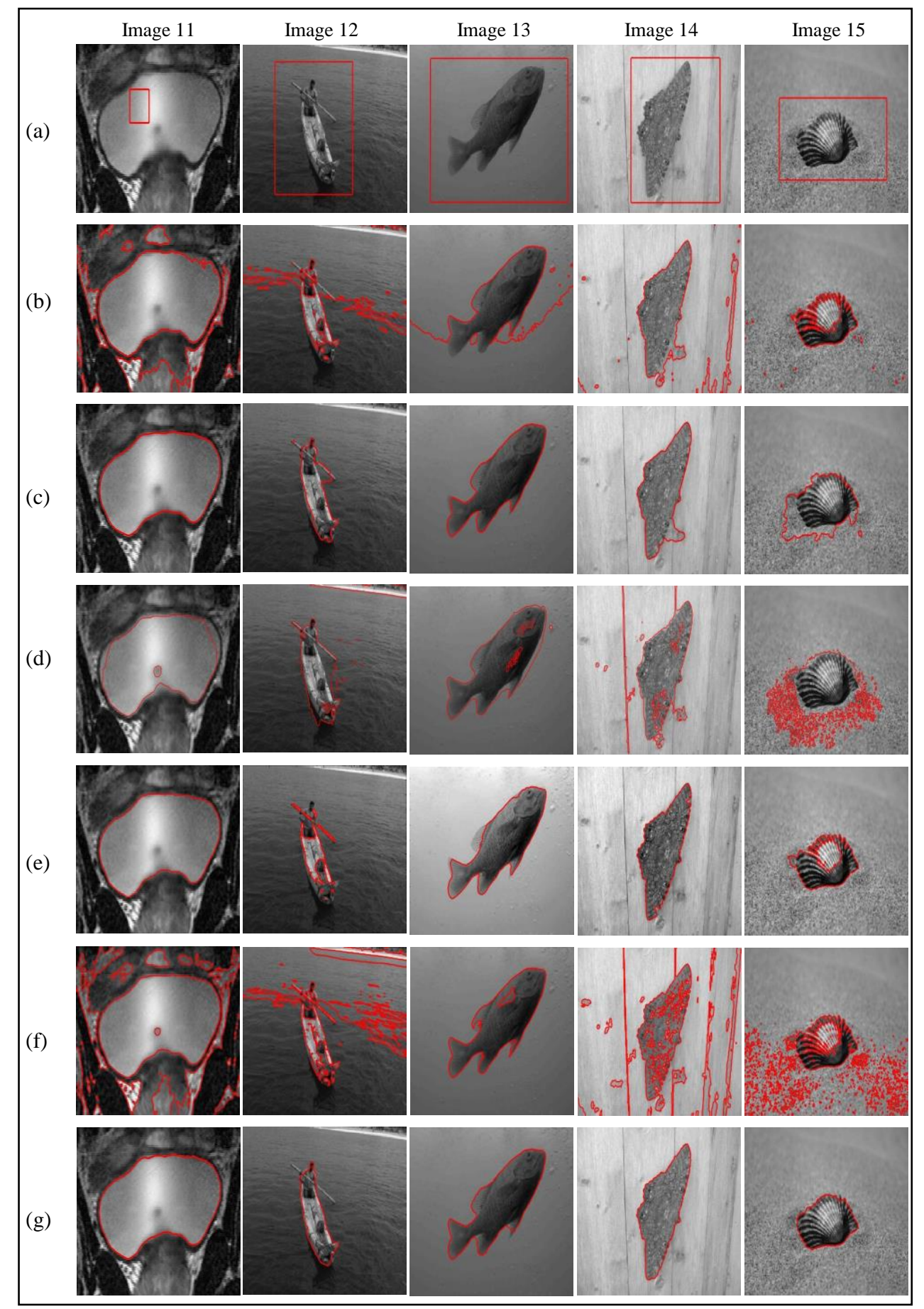

Fig. 7. Comparison between the different active contour models: Original images with initial contours (a). Segmentation output of: Gradient-based model [10] ( $\sigma=1.2$ for all images) (b), global region based model [17] (c), local region based model [21] ( $\rho=3$ for all images) (d), the hybrid models $[22,23](\mathrm{e}, \mathrm{f})$ and the proposed model $(\mathrm{g})$ 
For more comparison, some visualized LBPSF images of the set are contrasted with the ESF images in Fig. 8. Contrary to the ESF, it is clearly seen that the LBPSF can accurately distinguish the object boundaries.

Moreover, Fig. 9 shows the line profile plots for both LBPSF and ESF values across the hat area of image 8 . Here the noisy and poorly defined liver boundary (edge) along the profile is located at the pixel number "15"; unlike the ESF, the value of the LBPSF is exactly " 0 " on the edge and " 1 " in the two regions beside it. The graph shows that LBPSF is a robust indicator of poorly defined or noisy edges.
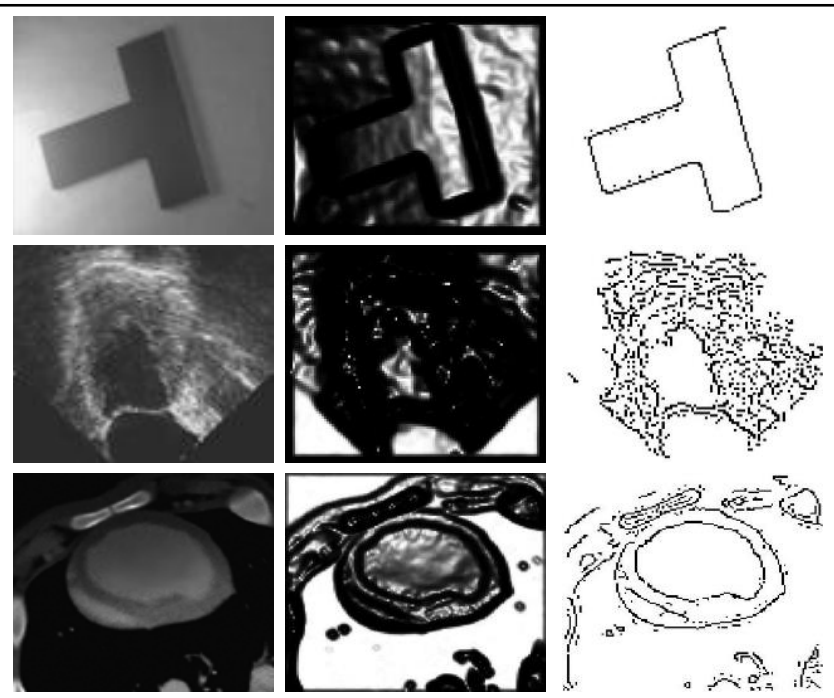

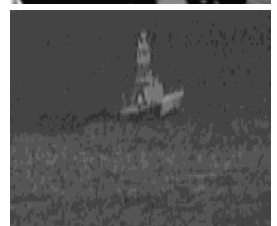

(a)

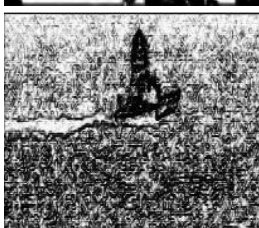

(b)

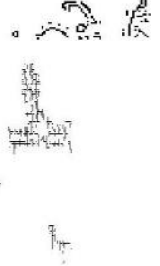

(c)

Fig. 8. Comparison between the ESF and the LBPSF: Original images (a), the visualized ESF of the original images (b), and the visualized LBPSF of the original images (c)

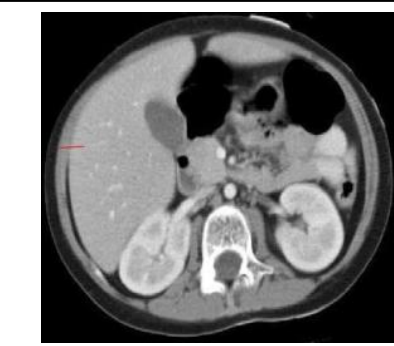

(a)

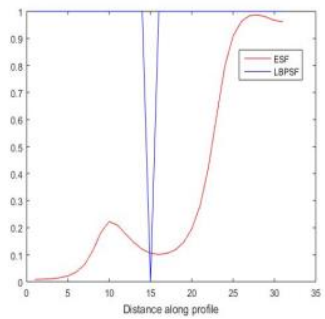

(b)

Fig. 9. Comparison between the ESF and the LBPSF: Original liver CT image (a), ESF and LBPSF values along a line (red) segment in the original image (b) 
Here the proposed model outperforms the model proposed in [17] since the set of images used requires local segmentation property, and the global region based ACMs generally suffer from intensity inhomogeneity problem.

Comparing the proposed model to the model proposed in [21] which gives closely similar results to those obtained by our model on images with several intensity levels (Image 2) or with intensity inhomogeneity (Images: 1, 6 and 7), this model seems to be sensitive to noise (Images: 3-5, 10 and 15) and the segmentation result relies on the size of the region scale parameter ( $\rho$ ) (Images: 8, 9 and 11-14).

To show the sensitivity of this model to region scale parameter $(\rho)$, we used images: 3, 6, 7 and 9 with three different sizes of $\rho$ (1.5, 3.0, and 6.0). Fig. 10 shows that depending on the image, small value of $\rho$ leads to local segmentation when the intensity inhomogeneity is severe (second and third row) while large value is chosen in noisy and smooth regions (first and last row).

Here the proposed model outperforms also the hybrid models proposed in [22] and [23]. Although, combining edge information or local region information with global region information raises to some extent the performance of the model (Images: 1, 3, 4, 6, 10, 13 and 14 for the model of [22] and Images 3 and 13 for the model of [23]) but, since the weighting parameter is a fixed positive constant and not chosen dynamically for each pixel, the segmenting curve is influenced by global region information too great, consequently, it got an incorrect result.

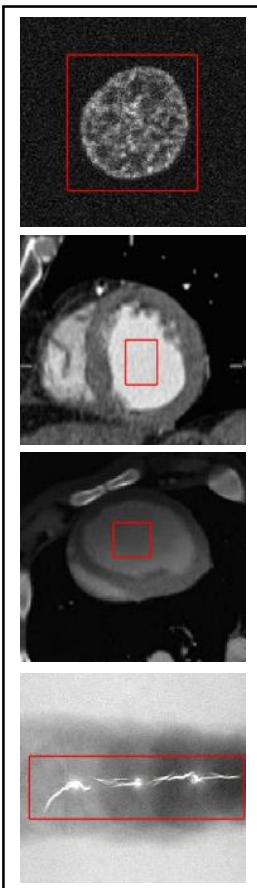

(a)
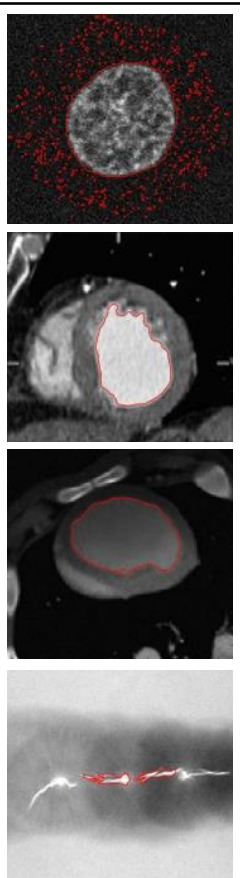

(b)
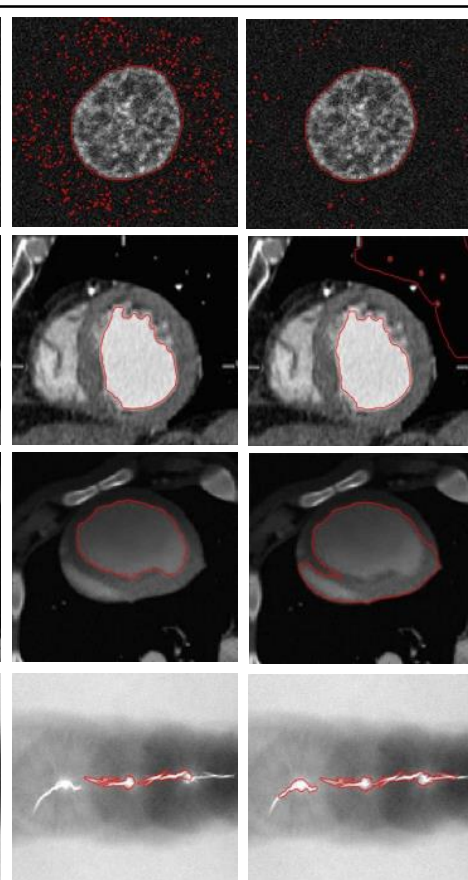

(c)
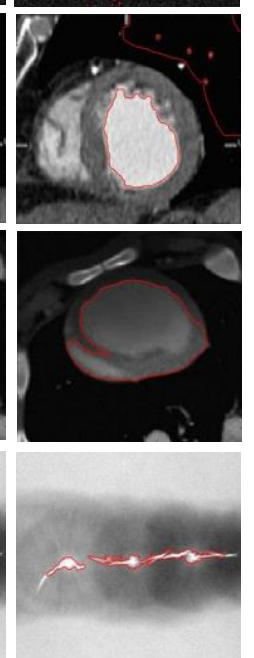

(d)

Fig. 10. Segmentation results by the model of [21]: Original images with initial contours (a), the segmentation results with $\rho=1.5, \rho=3.0$ and $\rho=6.0$, (b), (c) and (d), respectively 
Moreover the Dice coefficient [35] is used to compare and measure the segmentation accuracy. The Dice index $D \in[0,1]$ between the obtained segmentation result $R_{R}$ and the ground truth $R_{G}$ is given by: $D\left(R_{R}, R_{G}\right)=\frac{2 \operatorname{Area}\left(R_{R} \cap R_{G}\right)}{\text { Area }\left(R_{R}\right)+\operatorname{Area}\left(R_{G}\right)}$. A higher Dice value (close to 1 ) indicates better segmentation performance.

From the quantitative performance measure showed in Table 2 and Fig. 11, we observe that the proposed model yields almost the best segmentation accuracy with an average Dice score of 0.96, compared to $0.51,0.897,0.898,0.83$ and 0.55 for the models proposed in $[17,10,21,22,23]$ respectively. This accuracy of the proposed method caused by using the total energy defined in (9) that is based on the LBPSF has two major advantages:

- The LBPSF is constructed so that noise in image can be removed without affecting object boundaries due to the classification of LBP textons used in the Canny's edge detector based LBP.

- The non-maximum suppression step which is an edge-thinning algorithm has the property of thinning the edges; so that, the curve will stop at real object boundaries.

Computationally, we have not mentioned computation time in this study since the related methods and the proposed one use different level set strategies (PDE based LSM for the model proposed in [17] and variational LSM for the other models including the proposed one). More precisely, the number of iterations of the model proposed in [17] ranges from 5 to 7 iterations, from 5 to 100 iterations for the model proposed in [22], while for other models including the proposed one, the range of these values from 200 to 600 iterations over the set of images used.

Table 2. Values of the Dice index $D$ of the different models used in the study (Fig. 5 and Fig. 6)

\begin{tabular}{|c|c|c|c|c|c|c|}
\hline Image & $\begin{array}{c}D \text { of the } \\
\text { nodel of [17] }\end{array}$ & $\begin{array}{c}D \text { of the } \\
\text { nodel of [10] }\end{array}$ & $\begin{array}{c}D \text { of the } \\
\text { nodel of [21] }\end{array}$ & $\begin{array}{c}D \text { of the } \\
\text { nodel of [22] }\end{array}$ & $\begin{array}{c}D \text { of the } \\
\text { nodel of [23] }\end{array}$ & $\begin{array}{c}D \text { of the proposed } \\
\text { model }\end{array}$ \\
\hline Image 1 & 0.6 & 0.96 & 0.992 & 0.97 & 0.59 & $\mathbf{0 . 9 9 9}$ \\
\hline Image 2 & 0.5 & 0.98 & 0.997 & 0.5 & 0.66 & $\mathbf{0 . 9 9 9}$ \\
\hline Image 3 & 0.90 & 0.992 & 0.997 & 0.979 & 0.929 & $\mathbf{0 . 9 9 9}$ \\
\hline Image 4 & 0.3 & $\mathbf{0 . 9 6 8}$ & 0.939 & 0.927 & 0.28 & 0.961 \\
\hline Image 5 & 0.53 & 0.87 & 0.9 & 0.79 & 0.51 & $\mathbf{0 . 9 7}$ \\
\hline Image 6 & 0.4 & 0.97 & $\mathbf{0 . 9 9}$ & 0.95 & 0.62 & 0.972 \\
\hline Image 7 & 0.64 & 0.88 & $\mathbf{0 . 9 9 7}$ & 0.76 & 0.62 & 0.996 \\
\hline Image 8 & 0.43 & 0.96 & 0.88 & 0.85 & 0.43 & $\mathbf{0 . 9 6 1}$ \\
\hline Image 9 & 0.05 & 0.77 & 0.776 & 0.73 & 0.05 & $\mathbf{0 . 8 2}$ \\
\hline Image 10 & 0.19 & 0.52 & 0.68 & 0.81 & 0.43 & $\mathbf{0 . 9 3}$ \\
\hline Image 11 & 0.82 & 0.988 & 0.95 & 0.988 & 0.77 & $\mathbf{0 . 9 9 0}$ \\
\hline Image 12 & 0.16 & 0.87 & 0.76 & 0.57 & 0.17 & $\mathbf{0 . 9 2}$ \\
\hline Image 13 & 0.48 & 0.957 & 0.948 & 0.943 & 0.951 & $\mathbf{0 . 9 7 4}$ \\
\hline Image 14 & 0.85 & 0.958 & 0.89 & 0.978 & 0.68 & $\mathbf{0 . 9 8}$ \\
\hline Image 15 & 0.71 & 0.83 & 0.77 & 0.78 & 0.53 & $\mathbf{0 . 9 3}$ \\
\hline
\end{tabular}




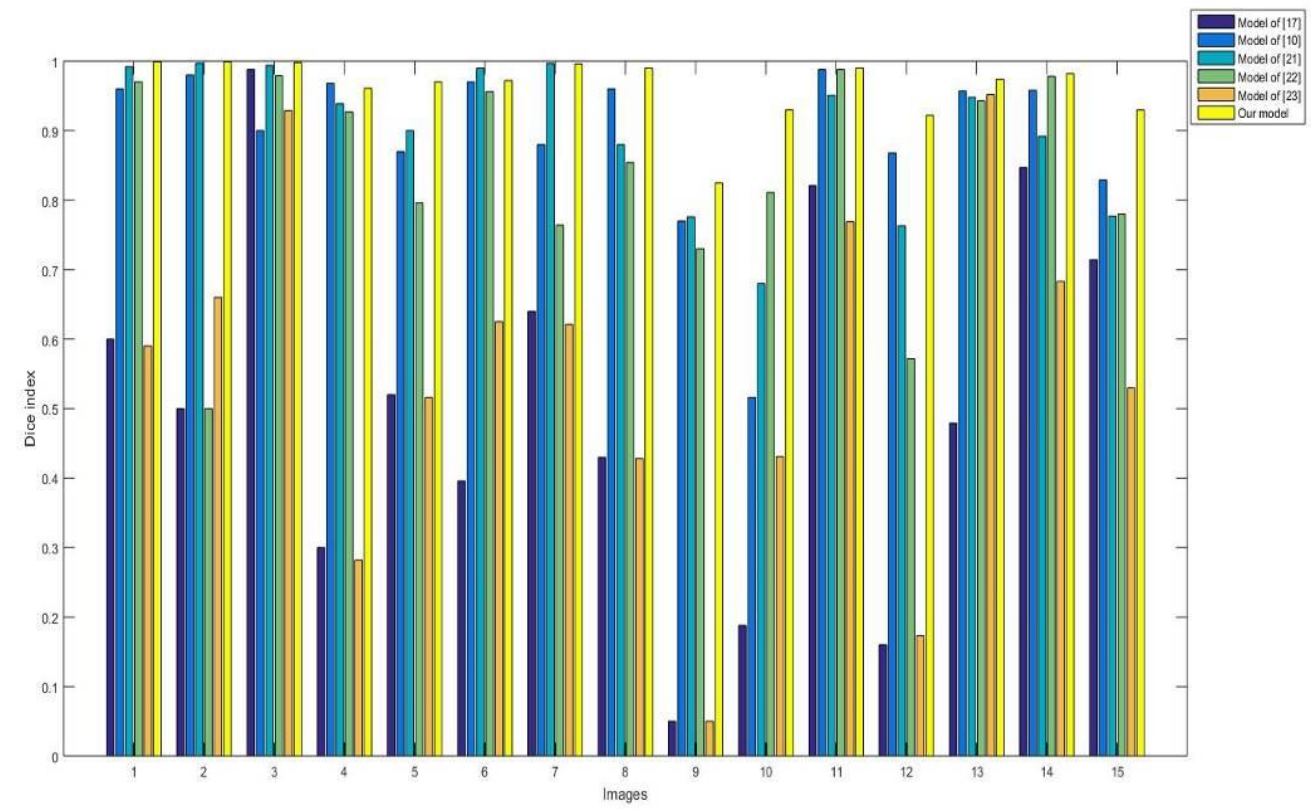

Fig. 11. Segmentation accuracy as calculated via the Dice index for each model

\section{Conclusion}

In this paper, a robust and efficient active contour model has been proposed by combining the local binary pattern stopping function (LBPSF) with the active contour model of [10]. Comparatively to edge stopping function (ESF), the LBPSF accurately distinguishes the noisy and poorly defined boundaries or edges. Experimental results and quantitative analysis showed that our method outperforms the related methods in terms of boundary detection and segmentation accuracy. Future work will be devoted to improve the performance of the proposed model by defining the threshold parameters in the hysteresis-thresholding step automatically, also to use the local binary patterns to adapt the size of the region scale parameter in local region based models.

Acknowledgement: We would like to thank Dr. Michael Teutsch for helping us to implement the edge detection method proposed in [28].

\section{References}

1. Ben Ayed, I., A. Mitiche. A Region Merging Prior for Variational Level Set Image Segmentation. - IEEE Transactions on Image Processing, Vol. 17, 2008, No 12, pp. 2301-2311.

2. Melouah, A., S. Layachi. A Novel Automatic Seed Placement Approach for Region Growing Segmentation in Mammograms. - In: Proc. of International Conference on Intelligent Information Processing, Security and Advanced Communication, Batna, Algeria, 2015, pp. 273-277.

3. K a s s, M., A. W i t ki n, D. Te r z o p o u lo s. Snakes. Active Contour Models. - International Journal of Computer Vision, Vol. 1, 1987, No 4, pp. 321-331. 
4. X u, C., J. Pri n c e. Snakes, Shapes, and Gradient Vector Flow. - IEEE Transactions on Image Processing, Vol. 7, 1998, No 3, pp. 359-369.

5. W u, Y., Y. W a n g, Y. J i a. Adaptive Diffusion Flow Active Contours for Image Segmentation. Computer Vision and Image Understanding, Vol. 117, 2013, No 10, pp. 1421-1435.

6. Cas elles, V., F. Catté, T. Coll, F. Dibos. A Geometric Model for Active Contours in Image Processing. - Numer. Math, Vol. 66, 1993, No 1, pp.1-31.

7. C a s e 11 e s, V., R. K i m m e 1, G. S a p i r o. Geodesic Active Contours. - International Journal of Computer Vision, Vol. 22, 1997, No 1, pp. 61-79.

8. Malladi, R., J. A. S e thi an, B. C. Ve muri. Shape Modeling with Front Propagation: A Level Set Approach. - IEEE Transactions on Pattern Analysis Machine Intellegence, Vol. 17, 1995, No 2, pp. 158-175.

9. Kumar, A., P. Olver, A. J. Yezzi, S. Kichenassamy, A. Tannenbaum. A Geometric Snake Model for Segmentation of Medical Imagery. - IEEE Trans. Med. Imag., Vol. 16, 1997, No 2, pp. 199-209.

10. Li, C., C. X u, C. G u i, M. D. F o x. Distance Regularized Level Set Evolution and Its Application to Image Segmentation. - IEEE Trans. Image Process, Vol. 19, 2010, No 12, pp. 3243-3254.

11. $\mathrm{Ch}$ a n, T., L. V e s e. Active Contours without Edges. - IEEE Trans. Image Process, Vol. 10, 2001, No 2, pp. 266-277.

12. Kimi a, B. B., A. Tannenbaum, S. Zucker. Shapes, Shocks, and Deformations I: The Components of Two dimensional Shape and the Reaction-Diffusion Space. - International Journal of Computer Vision, Vol. 15, 1995, No 3, pp. 189-224.

13. O s h e r, S., J. A. S e th i an. Fronts Propagating with Curvature-Dependent Speed: Algorithms Based on Halilton-Jacobi Formulations. - Journal of Computational Physics, Vol. 79, 1988, No 1, pp. 12-49.

14. K s a n t in i, R., B. B o u f a m a, S. J. M e m a r. A New Efficient Active Contour Model without Local Initializations for Salient Object Detection. - EURASIP Journal on Image and Video Processing, Vol. 40, 2013.

15. A z i zi, A., K. E 1 k o u rd. Fast Region-Based Active Contour Model Driven by Local Signed Pressure Force. - ELCVIA Electronic Letters on Computer Vision and Image Analysis, Vol. 15, 2016, No 1, pp.1-13.

16. L a n kt o n, S., A. T a n n e n b a u m. Localizing Region-Based Active Contours. - IEEE Trans. Image Process, Vol. 17, 2008, No 11, pp. 2029-2039.

17. T a l u, M. F. ORACM: Online Region-Based Active Contour Model. - Expert Systems with Applications, Vol. 40, 2013, No 16, pp. 6233-6240.

18. Li, C., C. K a o, J. G o r e, Z. D in g. Implicit Active Contours Driven by Local Binary Fitting Energy. - In: Proc. of IEEE Conf. Computer Vision and Pattern Recognition, Minneapolis, 2007, pp. 1-7.

19. Li u, S., Y. P e n g. A Local Region-Based Chan-Vese Model for Image Segmentation. - Pattern Recognition, Vol. 45, 2012, No 7, pp. 2769-2779.

20. Li, C., R. Hu ang, Z. Ding, J. C. Gatenby, D. N. Met ax as, J. C. Gore. A Level Set Method for Image Segmentation in the Presence of Intensity Inhomogeneities with Application to MRI. - IEEE Trans. Image Process, Vol. 20, 2011, No 7, pp. 2007-2016.

21. Z h a n g, K., L. Z h a n g, K-M. L a m, D. Z h a n g. A Level Set Approach to Image Segmentation with Intensity Inhomogeneity. - IEEE Transactions on Cybernetics, Vol. 46, 2016, No 2, pp. 546-557.

22. Z h a n g, Y., B. J. M a t u s z e w s k i, L.-K. S h a r k, C. J. M o o r e. Medical Image Segmentation Using New Hybrid Level-Set Method. - In: Proc. of 5th International Conference BioMedical Visualization Information Visualization in Medical and Biomedical Informatics, 2008, pp. 71-76.

23. W a n g, L., C. Li, Q. S u n. Active Contours Driven by Local and Global Intensity Fitting Energy with Application to Brain MR Image Segmentation. - In: Comput. Med. Imaging Graph, Vol. 33, 2009, No 7, pp. 520-531.

24. Azizi, A., K. Elkourd. A Hybrid Active Contour without Re-Initialization. - In: Proc. of International Conference on Intelligent Information Processing, Security and Advanced Communication, Batna, Algeria, 2015, pp. 239-244. 
25. Z h a n g, K., L. Zh a n g, H. S o n g, D. Zh a n g. Reinitialization-Free Level Set Evolution via Reaction Diffusion. - IEEE Trans. Image Process, Vol. 22, 2013, No 1, pp. 258-271.

26. Ojala, T., M. Pi etikä in en, T. Mä en ää. Multiresolution Gray-Scale and Rotation Invariant Texture Classification with Local Binary Patterns. - IEEE Transactions on Pattern Analysis and Machine Intelligence, Vol. 24, 2002, pp. 971-987.

27. Mä en pää, T. The Local Binary Pattern Approach to Texture Analysis - Extensions and Applications. - Dissertation, University of Oulu, Finland, 2003.

28. T e u t s c h, M., J. B e y e r e r. Noise Resistant Gradient Calculation and Edge Detection Using Local Binary Patterns. - In: Lecture Notes in Computer Science (LNCS). Vol. 7728. Computer Vision - ACCV 2012 Workshops. Berlin, Heidelberg, Springer, 2013, pp. 1-14.

29. A $1 \mathrm{t}$ a r a w n e h, N. M., S. Lu o, B. R e g a n, C. S u n. A Modified Distance Regularized Level Set Model for Liver Segmentation from Ct Images. - Signal and Image Processing, Vol. 6, 2015, No 1, pp. 1-11.

30. Z h a ng, K., L. Z h an g, H. S o n g, W. Z h o u. Active Contours with Selective Local or Global Segmentation: A New Formulation and Level Set Method. - Image and Vision Computing, Vol. 28, 2010, No 4, pp. 668-676.

31. A b d e $1 \mathrm{~s} \mathrm{a} \mathrm{m} \mathrm{e} \mathrm{a,} \mathrm{M.} \mathrm{M.,} \mathrm{G.} \mathrm{G} \mathrm{n} \mathrm{e} \mathrm{c} \mathrm{c} \mathrm{o,} \mathrm{M.} \mathrm{M.} \mathrm{G} \mathrm{a} \mathrm{b} \mathrm{e} \mathrm{r,} \mathrm{E.} \mathrm{E} 1$ y a n. On the Relationship between Variational Level Set-Based and SOM-Based Active Contours. - Computational Intelligence and Neuroscience, 2015. 19 p. Article ID 109029.

32. Ahone n, T., A. Hadid, M. P i et ikä in en. Face Description with Local Binary Patterns: Application to Face Recognition. - IEEE Trans. Pattern Anal. Mach. Intell, Vol. 28, 2006, No 12, pp. 2037-2041.

33. M a n d a v a, A. K., E. E. R e ge n t o v a. Speckle Noise Reduction Using Local Binary Pattern. In: Proc. of 2nd International Conference on Communication, Computing \&Amp; Security, Vol. 6, 2012, pp. 574-581.

34. Te ut s ch, M., P. Trantelle, J. B e yere r. Adaptive Real-Time Image Smoothing Using Local Binary Patterns and Gaussian Filters. - In: Proc. of 20th IEEE International Conference on Image Processing, Melbourne, 2013, pp. 1120-1124.

35. Mukherjee, S., S. T. Acton. Region Based Segmentation in Presence of Intensity Inhomogeneity Using Legendre Polynomials. - IEEE Signal Processing Letters, Vol. 22, 2015, No 3, pp. 298-302. 